\title{
IMAGES OF THE RESPONSE SIGNAL OF A 2D GAS OF CARRIERS TO A PULSED BEAM OF 3D PHONONS
}

\author{
Cz. Jasiukiewicz ${ }^{a}$, D. LehmanN ${ }^{b}$ AND T. PASzKIEWICZ ${ }^{a}$ \\ ${ }^{a}$ Institute of Theoretical Physics, University of Wrocław \\ Pl. M. Borna 9, 50-204 Wrocław, Poland \\ ${ }^{b}$ Technische Universität Dresden, Institut für Theoretische Physik \\ Mommsenstrasse 13, 0-8027 Dresden, Germany
}

The patterns for the time integrated drag current induced by a pulsed beam of bulk phonons in a $2 \mathrm{D}$ gas of charge carriers are calculated. A beam of Planckian phonons propagates in a GaAs crystal. We considered a $2 \mathrm{D}$ gas of electrons lying in a $\{001\}$ plane and a $2 D$ gas of holes lying in the $\{311\}$ plane. Planckian phonons are radiated by an extended (Gaussian) source.

PACS numbers: $63.20 . \mathrm{Kr}, 66.70 .+\mathrm{f}, 73.40 . \mathrm{Lq}$

\section{Introduction}

The interaction of a 2D electron gas (2DEG) and a 2D hole gas (2DHG) with bulk acoustic phonons has been studied by different techniques in recent years (for a review cf. [1] and [2]). Due to the reduced dimensionality of a 2D gas of charge carriers (2DCG), the interaction with bulk phonons differs considerably from their interaction with 3DCGs. Another interesting feature of a 2DCG is the smallness of the Fermi wave vectors $k_{\mathrm{F}}$ (and consequently the smallness of the Fermi momentum, the Fermi velocity $v_{F}$, and the Fermi temperature $T_{F}$ ). For a 2DCG the Fermi wave vector is comparable or even less than the typical wave vector of small energy acoustic phonons. Using phonon beams one can experimentally sweep relatively large volume of the $2 \mathrm{D}$ momentum space.

There are very practical reasons for doing experiments of this type, because the electron-phonon interaction plays an important role in limiting the mobility, i.e. the performance of low-dimensional devices. Collisions with phonons limit the room temperature mobility of electrons in 2D high electron mobility transistors (HEMTs). In a series of papers (cf. [2]) Dietsche and collaborators (the Stuttgart group - SG) published the results of measurements of the time integrated response of a 2DEG to pulsed beams of acoustic phonons. In their experiments phonons propagated in a GaAs crystal and interacted with the $2 \mathrm{D}$ electrons lying in a $\{001\}$ plane. They used sources of monochromatic phonons (a superconducting $\mathrm{Al}$ 
film heated by a pulsed laser beam, a superconducting tunnel junction) and also sources of thermal phonons (a metallic film heated by a pulsed laser beam). If the 2DCG structure is a part of an open circuit, phonons generate the transient voltage and for a short circuit - the transient electric current. There is no experimental evidence for a difference between patterns of the induced voltage and the induced current (cf. [2]).

In our previous papers we proposed a kinetic description of phonon focusing [3] and of the response of a 2DEG to a short duration pulse of monochromatic phonons, generated by a point source [4]. We noticed also a close relation of this response to the phenomenon of phonon quasimomentum focusing. For a disturbed 2DEG we calculated the time integrated induced current density (TICD) and studied it for different directions of the phonon propagation. For the selected directions studied experimentally (cf. [2]) we studied also the dependence of the time TICD on the phonon frequency $\omega$. We assumed that a source of phonons is fixed and the detector is movable (such experiments were performed by the Kaplyanskii group who used as a detector a cloud of excitons, and by the Challis group who used CdS detectors [1]). However, this method of performing measurements is completely equivalent to the method using movable sources and fixed detectors. Scanning the surface in which a 2DEG structure (a bridge) is lying, we calculated a set of TICD for selected propagation directions. The results, together with their coordinates, were stored in the memory of a computer. In order to obtain the image of a calculated TICD, the results of calculations were displayed on the monitor and also printed as grey tones.

The method of calculation is described in this issue [5]. We accounted for the finite dimensions of the source and of the detector and of a finite lifetime. According to Tamura [6] for the natural concentration of $\mathrm{Ga}$ isotopes $\tau_{\mathrm{GaAs}}^{-1}=$ $(2 \pi)^{4} \times 7.38 \times 10^{-41} \omega^{4}$.

\section{Patterns of phonon induced drag current}

The detector of quasimomentum - here a 2DCG structure (a bridge), has an elongated form and is directed along the vector $\hat{\eta}$. The normal to this structure is $\widehat{n}$.

The orientation of the 2DHG bridge used in the Nottingham group (NG) experiment is not known. Using a fast Monte Carlo simulation program developed by our group we generated a series of quasimomentum density patterns for different orientations of the bridge. In this way we concluded that $\widehat{\eta}=[0 \overline{1} 1]$ (cf. Table I). The suitable TICD pattern is calculated for this direction of the bridge.

The data related to the Stuttgart [2], the Nottingham [7] experiments are collected in Table I. Characteristics of the 2DCG studied by the above groups are summarized in Table II.

The calculated images of the TICD are shown in Fig. 1 (2DEG) and Fig. 2 (2DHG). We compared them to the corresponding experimentally obtained patterns $[2,7]$. The agreement is quite satisfactory. 
Characteristics of the experiments.

TABLE I

\begin{tabular}{c|c|c|c|c|c|c|c}
\hline \hline Group & $\hat{n}$ & $\hat{\eta}$ & $\begin{array}{c}\text { Specimen } \\
\text { thickness } \\
{[\mu \mathrm{m}]}\end{array}$ & $\begin{array}{c}\text { Source } \\
\text { dimensions } \\
{[\mu \mathrm{m}]}\end{array}$ & $\begin{array}{c}\text { Detector } \\
\text { dimensions } \\
{[\mu \mathrm{m}]}\end{array}$ & $\begin{array}{c}\text { Heater } \\
\text { temperature } \\
{[\mathrm{K}]}\end{array}$ & $\begin{array}{c}\text { Charge } \\
\text { density } \\
{\left[\mathrm{m}^{-2}\right]}\end{array}$ \\
\hline$(\mathrm{SG})[2]$ & {$[001]$} & {$[110]$} & 350 & 10 & $50 \times 50$ & $15-17$ & $3.76 \times 10^{15}$ \\
\hline$(\mathrm{NG})[7]$ & {$[311]$} & {$[0 \overline{1} 1]$} & 400 & $100 \times 100$ & $100 \times 100$ & 15 & $1.6 \times 10^{15}$ \\
\end{tabular}

Characteristics of the specimens.

\begin{tabular}{c|c|c|c|c|c|c}
\hline \hline Group & $\begin{array}{c}\text { Type } \\
\text { of } \\
\text { carriers }\end{array}$ & $\begin{array}{c}\text { Effective } \\
\text { mass } \\
\text { in } m_{\mathrm{e}}\end{array}$ & $\begin{array}{c}\text { Fermi } \\
\text { temperature } \\
T_{\mathrm{F}}[\mathrm{K}]\end{array}$ & $\begin{array}{c}\text { Fermi wave } \\
\text { vector } k_{\mathrm{F}} \\
10^{6}\left[\mathrm{~cm}^{-1}\right]\end{array}$ & $\begin{array}{c}\text { Fermi velocity } \\
v_{\mathrm{F}} \\
10^{4}\left[\mathrm{~ms}^{-1}\right]\end{array}$ & $\begin{array}{c}p= \\
2 e h_{14} \Xi_{\mathrm{D}}^{-1} \\
{\left[\mathrm{~cm}^{-1}\right]}\end{array}$ \\
\hline (SG) [2] & electrons & 0.067 & $1.41 \times 10^{2}$ & 1.54 & $2.6 \times 10^{1}$ & $9.7 \times 10^{6}$ \\
\hline (NG) [7] & holes & $\mathbf{0 . 4 7}$ & 8.47 & 1 & 2.38 & $9.7 \times 10^{6}$
\end{tabular}

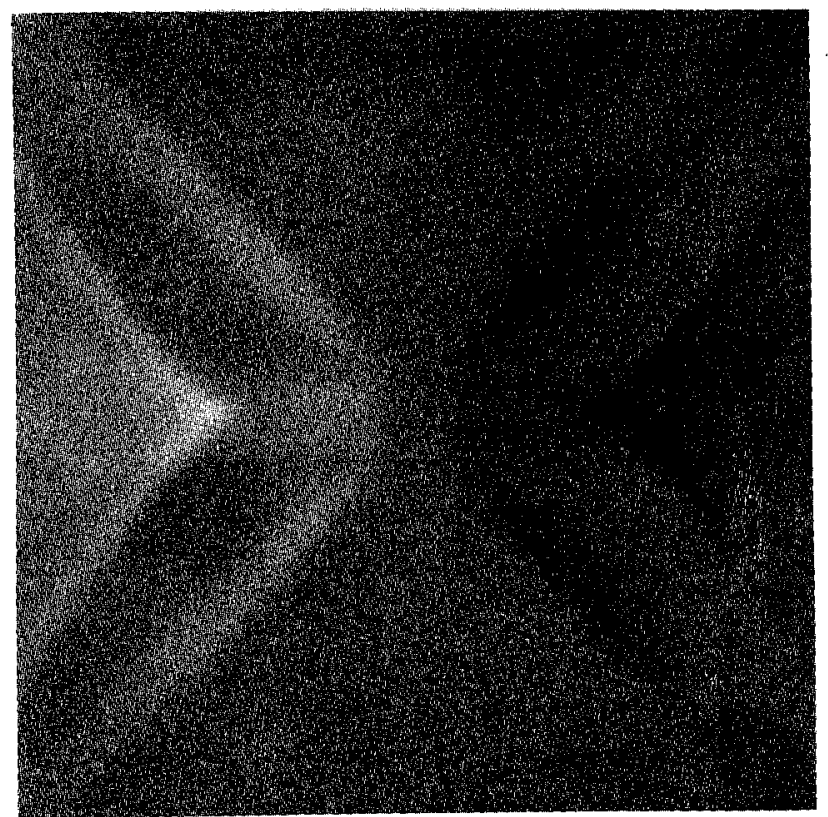

Fig. 1. The calculated pattern of the time integrated current density for a 2DEG for data of the Stuttgart group. Contributions of acoustic phonons of all three polarizations and both deformation potential and piezoelectric coupling are added.

The experimental pattern of the Nottingham group corresponds to the central part of the calculated pattern (Fig. 2). In both patterns one can distinguish caustics; however, in contrast to the calculated pattern the experimental pattern shows a pronounced asymmetry. The origin of this is not clear. 


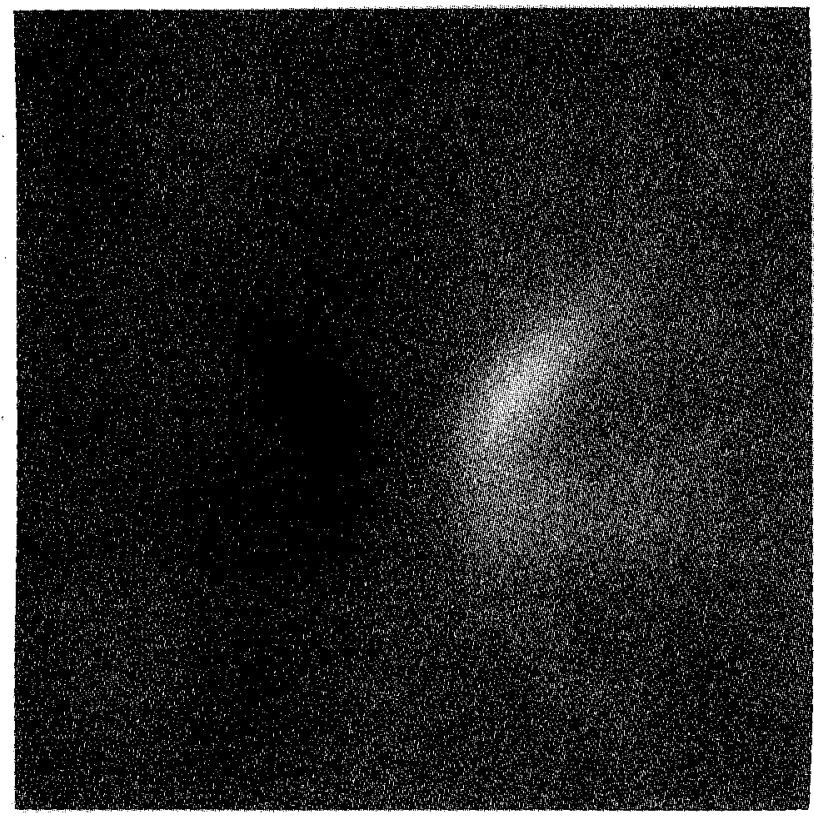

Fig. 2. The same as in Fig. 1 for a $2 D H G$ and the data of the Nottingham group.

\section{Acknowledgments}

This work is supported by a grant from the Committee for Scientific Research of Poland under the contract No. 397/2/91. We also acknowledge Werner Dietsche and Tony Kent who supplied their experimental patterns.

\section{References}

[1] L.J. Challis, A.J. Kent, in: Die Kunst of Phonons, Proc. XXIX Winter School of Theoretical Physics, Eds. T. Paszkiewicz, K. Rapcewicz, Plenum Press, New York 1993, in preparation; A.J. Kent, in: Phonon Scattering in Condensed Matter VII, Eds. M. Meissner, R.O. Pohl, Springer, Berlin 1993, p. 351.

[2] F. Dietzel, W. Dietsche, K. Ploog, in: Die Kunst of Phonons, Proc. XXIX Winter School of Theoretical Physics, Eds. T. Paszkiewicz, K. Rapcewicz, Plenum Press, New York 1993, in preparation.

[3] Cz. Jasiukiewicz, D. Lehmann, T. Paszkiewicz, Z. Phys. B, Condens. Matter 84, 73 (1991).

[4] Cz. Jasiukiewicz, D. Lehmann, T. Paszkiewicz, Z. Phys. B, Condens. Matter 86, 225 (1992).

[5] Cz. Jasiukiewicz, T. Paszkiewicz, Acta Phys. Pol. A 84, 459 (1993).

[6] S. Tamura, Phys. Rev. B 30, 849 (1984).

[7] T. Kent, private information, cf. also A.J. Kent, K.R. Strickland, M. Henini, in: Phonon Scattering in Condensed Matter VII, Eds. M. Meissner, R.O. Pohl, Springer, Berlin, p. 359. 